\section{BMJ Open Respiratory Research}

\title{
Deoxynucleoside therapy for respiratory involvement in adult patients with thymidine kinase 2-deficient myopathy
}

Ana Hernandez-Voth, ${ }^{1,2}$ Javier Sayas Catalan, ${ }^{1,2,3}$ Marta Corral Blanco, ${ }^{1,2}$ Alba Castaño Mendez, ${ }^{1}$ Miguel Angel Martin, ${ }^{3,4,5}$ Carlos De Fuenmayor Fernandez de la Hoz, ${ }^{2}$ Victoria Villena Garrido, ${ }^{3,6,7,8}$ Cristina Dominguez-Gonzalez $z^{2,3,9}$

\section{ABSTRACT}

Background Recessive mutations in the thymidinekinase 2 (TK2) gene cause a rare mitochondrial myopathy, frequently with severe respiratory involvement. Deoxynucleoside therapy is currently under investigation. Research question What is the impact of nucleosides in respiratory function in patients with TK2-deficient myopathy?

Study design and methods Retrospective observational study of patients treated with deoxycytidine and deoxythymidine. Evaluations were performed every 3 to 4 months after treatment during approximately 30 months. Forced vital capacity (FVC), maximuminspiratory and expiratory pressures (MIP/MEP), sniff nasal inspiratory pressure (SNIP), cough peak flow (CPF), arterial blood gas and nocturnal pulse oximeter (Sp02) were collected. Results We studied six patients, five of which were women, with a median age at onset of symptoms was 35.8 (range 5 to 60 ) years old. Patients presented a restrictive ventilatory pattern (median FVC of 50 (26 to 71)\%) and severe neuromuscular respiratory weakness (MIP 38 (12 to 47$) \%$ and SNIP 14 (8 to 19) $\mathrm{cmH20}$ ). Four patients required ventilatory support before starting the treatment. FVC improved by $6 \%$, proportion of sleep time with $\mathrm{Sp02}$ $<90 \%$ diminished from $14 \%$ to $0 \%$, CPF increased by $23 \%$, MEP increased by $73 \%$, production and management of bronchial secretions improved and respiratory infections diminished.

Interpretation Early detection of respiratory involvement requires an active search, even in asymptomatic patients. The nucleosides therapy may improve respiratory function, and stabilise the loss of respiratory capacity.

\section{Check for updates}

\section{(C) Author(s) (or their} employer(s)) 2020. Re-use permitted under CC BY-NC. No commercial re-use. See rights and permissions. Published by BMJ.

For numbered affiliations see end of article.

Correspondence to Dr Ana Hernandez-Voth; anarosario.hernandez@salud. madrid.org

\section{INTRODUCTION}

Late-onset thymidine kinase 2 (TK2)-deficient myopathy is a rare and only recently recognised disorder ${ }^{1}$ (MIM \#609560) that is caused by recessive mutations in the $T K 2$ gene.

TK2 deficiency predominantly manifests as a mitochondrial myopathy, with a broad spectrum of severity. It includes both extremely severe and rapidly progressive early-onset forms, with a survival of less than 2 years, and less severe forms with a childhood, late or very
Key messages

Can this new therapy with nucleosides actually improve and maintain respiratory function in adults with thymidine kinase 2-deficient myopathy?

The nucleosides therapy improves and stabilises respiratory function in adult patients with thymidine kinase 2-deficient myopathy.

These is the only paper describing the results of a new experimental therapy in respiratory function of a rare neuromuscular disease. Very few neuromuscular disorders have an effective treatment, and this could be one of them.

late onset, and a slower rate of progression, but with frequent respiratory involvement that shortens the patient's life expectancy. ${ }^{2-4}$

A detailed description of the late onset form of the disease has only been reported in a recently published series of 18 cases. ${ }^{3}$ This review suggests preferential effects on the diaphragm and other respiratory muscles. Indeed, respiratory insufficiency is the most common cause of death. Diaphragmatic weakness was identified in all cases reported in this series, as $66.6 \%$ of the patients required mechanical ventilation during the course of the disease. Diaphragmatic weakness was the cause of the first medical consultation in approximately half of the patients. Other symptoms included progressive proximal and distal limb muscle weakness, facial weakness, neck flexor weakness and axial weakness, that was often associated with isolated ptosis or ptosis with chronic external ophthalmoplegia. Neck flexor weakness was more severe than limb weakness.

The respiratory involvement of patients with neuromuscular disorders has generally been evaluated with standard spirometry, using forced vital capacity (FVC) as the 
main prognostic value. Nevertheless, since FVC can be preserved even with severe respiratory muscle weakness, ${ }^{5}$ additional respiratory tests may be necessary to improve accuracy and early detection of respiratory insufficiency. In addition, more sensitive tests are needed to detect minor positive changes during treatment, such as maximum inspiratory and expiratory pressures (MIP and MEP), sniff nasal inspiratory pressure (SNIP), cough peak flow (CPF) and nocturnal blood oxygenation (CT90) ${ }^{6-8}$

A new treatment for TK2 deficiency, based on the administration of oral deoxynucleosides, is currently under investigation. Its efficacy was reported in preclinical studies ${ }^{9-11}$ and recently in patients treated under a compassionate use/expanded access programme. Without any major side effects, the therapy exerted striking effects on patients with early-onset severe myopathy, produced considerable functional improvements in childhood-onset cases and at least stabilised late-onset cases. $^{12}$

Here, we describe a series of six adult patients with TK2-deficient myopathy who were treated under a compassionate use protocol with oral deoxythymidine $(\mathrm{dT})$ and deoxycytidine $(\mathrm{dC})$ and evaluated the response to treatment by focussing on the respiratory impairment. We analysed respiratory functional tests and non-invasive mechanical ventilation parameters by comparing the basal records with post-treatment evaluations.

\section{METHODS}

\section{Patients and public involvement}

Six adult patients with a genetic diagnosis of TK2 deficiency who were treated and followed in the Neuromuscular Unit of the 12 de Octubre University Hospital in Madrid, Spain, were included. Informed consent with study details was obtained from patients, and the written consent was gained from each enrolled subject. Abnormal results and the patient-reported symptoms were reviewed by the clinical principal investigator to determine the severity of the abnormality. The clinical investigator informed the participants if abnormalities were clinically significant. All but one of these patients (P6) have been described elsewhere. ${ }^{312}$

\section{Treatment}

All patients were treated with oral doses of both nucleosides (dT and dC), ranging from 200 to $400 \mathrm{mg} / \mathrm{kg}$ / day during an average period of 30 months (range 8 to 48 ) by the end of the data collection process. Doses administered to patients were based on the dosages used in preclinical studies of H126N Tk2 mutant mice ${ }^{29}$ and titrated depending on tolerance.

\section{Study design}

A retrospective observational study aimed at evaluating respiratory involvement in adult patients with a TK2 deficiency and analysing the respiratory effects of deoxynucleoside therapy. Data were collected between July 2015 and June 2019.

\section{Respiratory function tests}

Respiratory evaluations were performed before starting treatment and at different time points during follow-up (4, 7, 12, 18, 26 and 30 months after treatment). The following measurements of respiratory function were recorded in each visit: standard spirometry for FVC (Jaeger MasterScope, Carefusion, Hoechberg, Germany), MIP, MEP and SNIP (MicroRPM, Carefusion, Hoechberg, Germany), CPF (MicroPeak, Carefusion, Hoechberg, Germany), blood arterial gas for $\mathrm{pH}, \mathrm{paCO}_{2}$, $\mathrm{paO}_{2}$ and $\mathrm{HCO}_{3}(\mathrm{Gem}$ Premier 4000, Instrumentation Laboratory, Bedford, Massachusetts, USA), nocturnal pulse oximeter for $\mathrm{SpO}_{2}$ and CT90 (Pulsox 300i, Konica Minolta, Tokyo, Japan), a BiPAP (Bilevel Positive Airway Pressure) built in software assessment for IPAP (inspiratory positive airway pressure), EPAP (expiratory positive airway pressure), daily use of the device in hours and average number of spontaneous breaths (Philips DirectView, Murrysville, Pennsylvania, USA, and Resmed ResScan, Australia) and the need for mechanical cough assistance techniques (CoughAssist, Philips Respironics, Murrysville, Pennsylvania, USA). All respiratory function tests were performed according to international ATS/ ERS (American Thorax Society / European Respiratory Society) guidelines. ${ }^{13}$

\section{Non-invasive mechanical ventilation}

Patients were adapted to non-invasive mechanical ventilation if meeting the criteria of national guidelines and experts' recommendations on hypoventilation symptoms, orthopnoea, daytime hypercapnia, nocturnal hypercapnia or pathological results in lung function tests in neuromuscular diseases. ${ }^{1415}$

\section{Statistical methods}

For the analysis of the studied variables, a statistical software (SPSS 26, IBM) was used. Due to the small size of the sample, central and dispersion measurements were estimated using descriptive analyses with median and range, and a non-parametric test (Wilcoxon signed-rank) was used to evaluate the respiratory changes during the treatment in each patient. Statistical significance was defined as $\mathrm{p}<0.05$.

\section{RESULTS}

We studied six adult patients with a TK2 deficiency, five women and one man. The median age was 47.5 (range 31 to 73 years), and the median age at onset was 35.8 (range 5 to 60 ) years old.

General characteristics of these patients are listed in table 1 . 


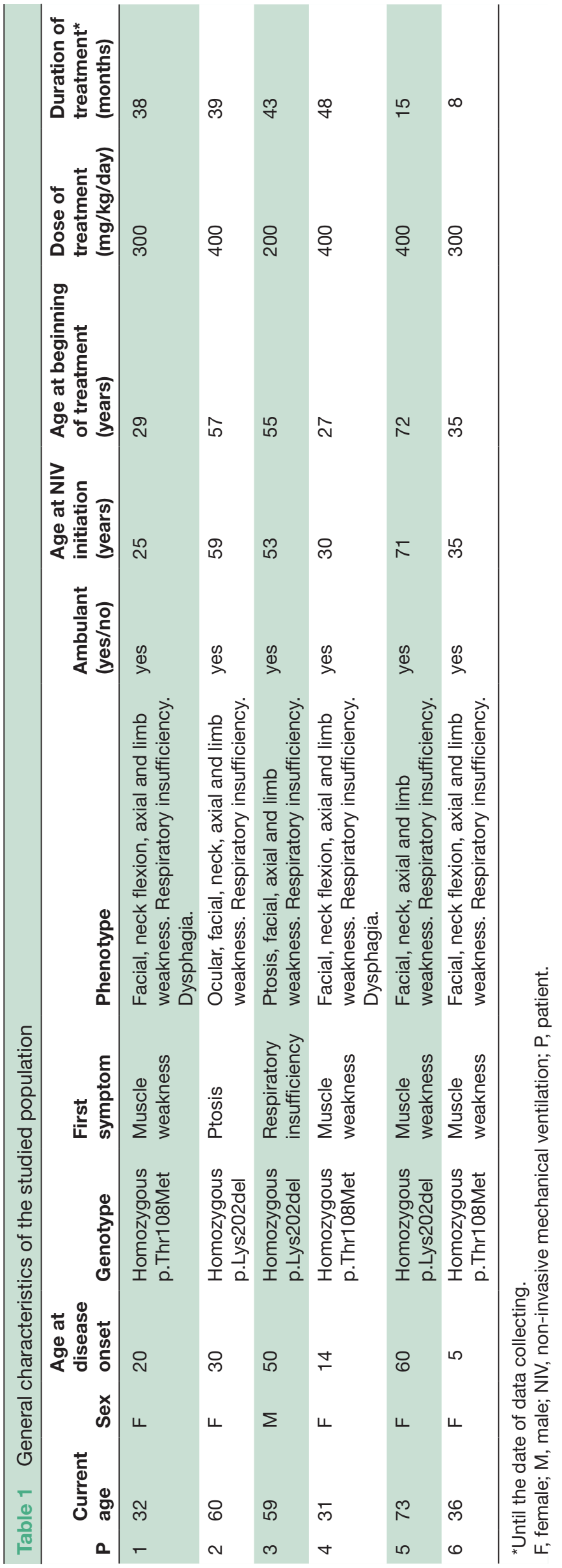

Respiratory function prior to treatment initiation

At baseline visit, patients presented a restrictive ventilatory pattern characterised by a substantial decrease in the FVC (median of 1780 (880 to 1920) mL, 50 (26 to 71)\% of the predicted value), MIP (median of 38 (12 to 47 ) $\%$ ) and SNIP values (median of 14 (8 to 19) $\mathrm{cmH} 2 \mathrm{O}$ ) (table 2).

At the time of starting the treatment, four of the six patients required ventilatory assistance with home noninvasive mechanical ventilation (NIV) (average usage of $7.75 \pm 2.06$ hours per day), two of them had a respiratory debut: one of them had developed an acute respiratory failure triggered by a respiratory tract infection (P3) and the other triggered by the anaesthesia required for a hip fracture surgical intervention (P5).

The two patients who were not on home NIV before starting treatment also presented respiratory symptoms at the time of the first neurological evaluation (orthopnoea, excessive daytime sleepiness, morning headache or drowsiness and unrestful sleep), suggesting diaphragmatic involvement and/or nocturnal hypoventilation.

\section{Other relevant clinical characteristics at baseline}

All patients experienced moderate muscle weakness involving the face, neck flexion (all patients were unable to lift the head while lying in the supine position) and proximal limb weakness with difficulties climbing stairs, rising to a standing position from the ground, jumping or running, but all patients were able to walk without assistance. Two patients had dysphagia: Patient 1 (P1) had a percutaneous gastrostomy tube placed due to recurrent pneumonias related to bronchoaspiration episodes and patient $4(\mathrm{P} 4)$ had a low body mass index.

\section{Respiratory evaluations during treatment}

Patients started treatment with $\mathrm{dT}+\mathrm{dC}$ for an average of 32 months by the end of the data collection period.

Table 2 as well as figure 1, show the results of the baseline respiratory function tests and their changes over time in patients receiving treatment.

After an average of 4 months of treatment, the median FVC improved both in terms of absolute volume $(+160 \mathrm{~mL}$ plus) and the percentage of reference values $(+5 \%$ increase). This improvement of FVC was maintained during the second evaluation (median of 7 months after treatment) and the third evaluation (median of 13 months after treatment) (table 2) and it showed statistical significance despite the small sample that we were analysing $(p<0.05)$. In the fifth and last evaluation (median of 30 months after treatment) the median FVC returned to the same value as the initial evaluation (table 2).

Nocturnal blood oxygenation (measured using nocturnal oximetry) improved. The median CT90 was reduced from 14 (6 to 21 ) \% to $3 \%$ in the first evaluation after treatment and the improvement persisted in subsequent evaluations (table 2). This recovery of nocturnal oxygenation was not only observed in patients with BiPAP 


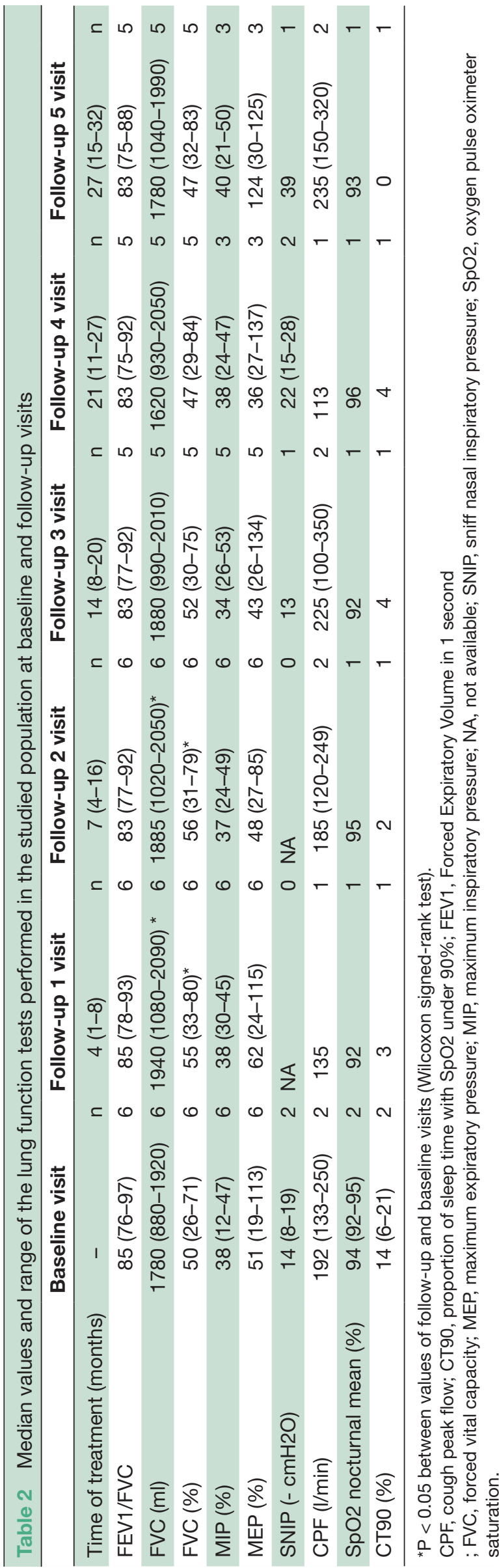

but also in a patient who was not yet receiving ventilatory support (Patient 2 (P2)), decreasing from 21\% before the treatment to $0 \%$ after a median of 30 months.

Expiratory muscle strength, assessed by CPF and MEP, exhibited a market but not statistically significant increase. After 12 months of treatment, CPF increased by $17 \%(+33 \mathrm{l} / \mathrm{min})$, and then continued to increase for up to a maximum of $23 \%$ above the baseline measurement $(+431 / \mathrm{min})$ after 26 months of treatment. The MEP increased from $51 \%$ to $124 \%$, reaching even normal values, after a median of 30 months of treatment (table 2). The production and management of bronchial secretions improved significantly. Prior to treatment, P1 and P3 required daily sessions of mechanically assisted cough techniques for the management of bronchial secretions, and after 6 and 11 months of treatment, respectively, the devices were no longer needed. The frequency and severity of respiratory infections also decreased. Prior to treatment P1, P2 and P3 presented repetitive bronchial infections, with frequent hospitalisation requirements. However, after 4 months of treatment, hospitalisations for respiratory infections were no longer necessary, and none were recorded after 18 months of treatment.

Arterial blood gas (ABG) samples were obtained, and its parameters (paCO2, paO2, $\mathrm{HCO} 3$ and $\mathrm{pH}$ ) remained stable throughout the follow-up period. Since most of the patients at the beginning, and all patients at the end of the study, received NIV which clearly affects ABG, these parameters probably do not show the progression of the disease, nor the evolution with the nucleosides therapy.

As we previously described, two patients had no home NIV before the treatment although they did have hypoventilation suggesting symptoms. However, after the initiation of treatment, the symptoms improved and NIV was not required to be initiated until 2 and 3 years after, respectively (P2 and $\mathrm{P} 4)$. No increase in ventilation requirements (hours of use or pressure support levels) was observed in any patient throughout the study (table 3).

\section{DISCUSSION}

A TK2 deficiency is a mitochondrial myopathy that is responsible for premature death in all its clinical forms, due to the severe, early and progressive involvement of the respiratory muscles. Recently, a deoxythymidine/ deoxycytidine substrate enhancement therapy showed very promising results, particularly in early-onset cases. In adults, the response to treatment remains unclear, and it may be limited by metabolic changes, as suggested in recent preclinical studies. ${ }^{11}$ Here, we describe a series of six adult patients focussing on respiratory involvement and its response to treatment.

In this series, TK2 deficiency caused respiratory impairments in all patients, who exhibited respiratory symptoms since the very beginning of the disease. Four of the six patients included in this series $(67 \%)$ were receiving respiratory support with NIV, two of them prior to the 
Figure 1. Evolution of principal lung function tests at baseline and after treatment

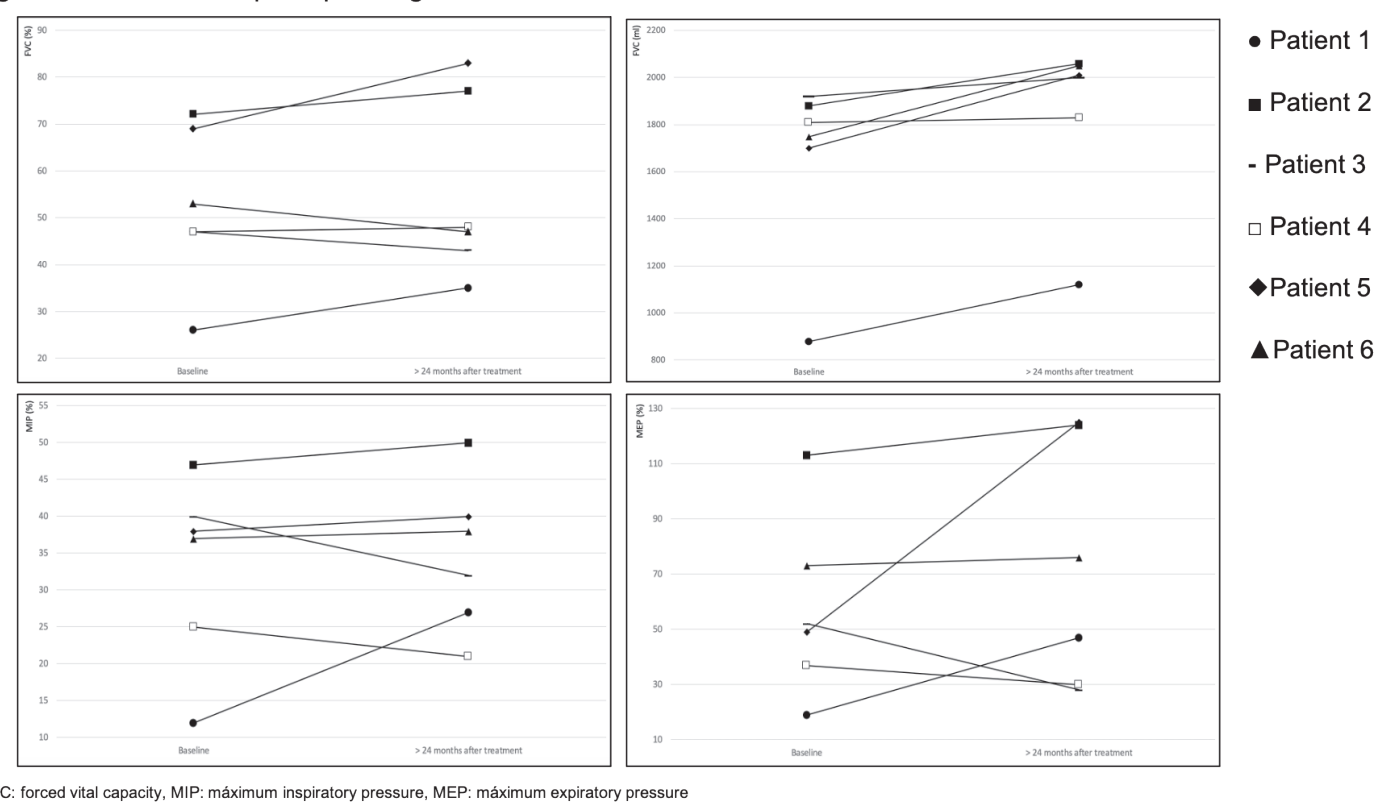

Figure 1 Evolution of principal lung function test at baseline and after treatment. FVC,forced vital capacity; MEP, maximumexpiratory pressure; MIP, maximum inspiratory pressure.

diagnosis of the TK2 deficiency and the other two prior to the initiation of treatment with deoxynucleosides. In the most detailed series reported to date describing a late-onset TK2 deficiency, ${ }^{3} 66 \%$ of patients required NIV during the course of the disease, and severe ventilatory insufficiency was the cause of the first consultation in $44 \%$ of patients. ${ }^{3}$ Eventually, all of our six patients required nocturnal NIV a few years after the diagnosis, even during the continuous deoxynucleoside therapy.

Interestingly, there is no correlation between diaphragmatic and limb weakness, and patients can suffer severe respiratory insufficiency in the presence of a mild limb muscle impairment. Therefore, the identification of signs of nocturnal hypoventilation is critical during the clinical evaluation of these patients, regardless of the severity of the skeletal myopathy as even patients with the ability to walk independently may require ventilatory support due to nocturnal hypoventilation.

According to spirometry tests, after starting the treatment, a relevant and statistically significant improvement in the FVC was noted, mainly during the first year of treatment. Other tests evaluating inspiratory muscle function, such as SNIP and MIP, also improved, although the differences were not statistically significant. After treatment, SNIP tripled its baseline value in 4 months, while MIP exhibited a slower but consistent improvement in 2years.

By the end of the follow-up period, all patients were treated with NIV, regardless of treatment with deoxynucleoside therapy, but no progression on the requirements for ventilatory support was noticed. This result reinforces the observation that respiratory functional performance improved or at least maintained its baseline status, but definitively did not decrease after 30 months of follow-up. In addition, we hypothesised that deoxynucleosides would have delayed the initiation of NIV treatment in the two nonventilated patients at baseline, since these patients already had symptomatic restrictive ventilatory dysfunction before starting treatment but did not need the ventilatory support until 2 and 3 years later, respectively.

After 1 year of treatment, the results of tests performed to evaluate expiratory muscle function improved notably. The CPF improved and MEP reached a normal value. These changes were clinically relevant, since frequency and severity of respiratory tract infections systematically decreased and the use of devices for mechanically cough assist were discontinued in two patients, but did not achieve statistical

\begin{tabular}{lcccccc}
\hline \multicolumn{2}{l}{ Table 3} & Median and range values of ventilatory & parameters and adherence to NIV & & \\
\hline Visit & Baseline & Follow-up 1 & Follow-up 2 & Follow-up 3 & Follow-up 4 & Follow-up 5 \\
\hline Time of treatment (months) & - & $4(1-8)$ & $7(4-16)$ & $13(7-20)$ & $21(11-27)$ & $30(21-33)$ \\
Patients requiring NIV (\#) & 4 & 4 & 4 & 4 & 4 & 6 \\
IPAP (cmH2O) & $17(14-17)$ & $17(14-18)$ & $16(14-8)$ & $16(14-18)$ & $16(15-17)$ & $17(14-19)$ \\
EPAP (cmH2O) & $5(5-6)$ & $6(5-6)$ & $6(5-6)$ & $6(5-6)$ & $6(5-7)$ & $7(5-8)$ \\
Use (hours/day) & $8(5-8)$ & $8(5-10)$ & $8(4-9)$ & $8(6-9)$ & $8(8-10)$ & $8(6-10)$ \\
\hline
\end{tabular}

EPAP, expiratory positive airway pressure; IPAP, inspiratory positive airway pressure; NIV, non-invasive mechanical ventilation. 
significance, probably due to the small size of the studied population.

We recognise important limitations of this study: (1) The absence of a control group and the small sample of patients included. However, late-onset TK2 deficiency is an ultrarare disease and this series represent a high proportion of adult patients treated worldwide. (2) The great variation in follow-up times and the absence of some important data (such as nocturnal transcutaneous capnography before the initiation of non-invasive mechanical ventilation), due to the fact that it is a retrospective study.

In summary, this study is the first one that specifically focusses on the respiratory impairment in adult patients with mitochondrial myopathy due to a TK2 deficiency. Without treatment, patients exhibit a high risk of early and progressive respiratory insufficiency that becomes the most common cause of death. Furthermore, a severe acute respiratory failure may be the first manifestation of the disease in a subset of patients. Early detection of respiratory involvement requires an active search in the clinics, even in patients with only mild limb weakness, as a preferential diaphragmatic involvement has been suggested. Deoxynucleoside therapy may improve the results of functional tests and diaphragmatic function in the short-term and stabilise the loss of respiratory capacity in the medium-term in patients with a TK2 deficiency. This finding is very relevant since respiratory insufficiency exerts substantial effects on the quality of life and survival, and is the main factor of poor prognosis in patients. Nevertheless, longitudinal studies with a longer follow-up period are needed to confirm the beneficial effects of the deoxynucleoside therapy on the respiratory function and survival of patients with a TK2 deficiency.

\section{Author affiliations}

${ }^{1}$ Servicio de Neumología, Unidad de Ventilación Mecánica, Hospital Universitario 12 de Octubre, Madrid, Spain

${ }^{2}$ Servicio de Neurologia, Unidad de Enfermedades Neuromusculares, Hospital Universitario 12 de Octubre, Madrid, Spain

${ }^{3}$ Instituto de Investigacion imas12, Hospital Universitario 12 de Octubre, Madrid, Spain

${ }^{4}$ Departamento de Bioquímica, Laboratorio de Enfermedades Mitocondriales, Hospital Universitario 12 de Octubre, Madrid, Spain

${ }^{5}$ Centro de Investigación Biomédica en Red de Enfermedades Raras, Instituto de Salud Carlos III, Madrid, Spain

${ }^{6}$ Departamento de Medicina, Facultad de Medicina, Universidad Complutense de Madrid, Madrid, Spain

${ }^{7}$ Centro de Investigación Biomédica en Red de Enfermedades Respiratorias (CIBERES), Instituto de Salud Carlos III, Madrid, Spain

${ }^{8}$ Servicio de Neumología, Hospital Universitario 12 de Octubre, Madrid, Spain

${ }^{9}$ Centro de Investigacion Biomedica en Red de Enfermedades Raras

(CIBERER), Instituto de Salud Carlos III, Madrid, Spain

Contributors All authors in this paper contributed to the design of the work, the acquisition, analysis and interpretation of data. All authors revised the work for intellectual content, and also, they all approved the submitted version.

Funding This work was supported by research grants of Spanish Instituto de Salud Carlos III (ISCIII), PMP15-00025 and PI18-01374 and the European Regional Development Fund (FEDER a way to achieve Europe) to MAM.

Competing interests $\mathrm{AH}-\mathrm{V}$ and $\mathrm{CD}-\mathrm{G}$ are currently involved in a clinical trial (NCT03845712) designed to evaluate safety and efficacy of deoxythymidine/ deoxythymidine substrate enhancement therapy in TK2-deficient myopathy patients (Sponsor: Modis Therapeutics, Inc). Nevertheless, we here present retrospective data of our experimentally treated patients, prior to the start of funded research.

Patient and public involvement Patients and/or the public were involved in the design, or conduct, or reporting, or dissemination plans of this research. Refer to the Methods section for further details.

Patient consent for publication Not required.

Ethics approval The institutional review board of the Hospital "Comite de Etica para la Investigación Clínica - CEIC" revised and approved the study under the number 16/070.

Provenance and peer review Not commissioned; externally peer reviewed.

Data availability statement All data relevant to the study are included in the article or uploaded as supplementary information. All data relevant to the study are included in the article.

Open access This is an open access article distributed in accordance with the Creative Commons Attribution Non Commercial (CC BY-NC 4.0) license, which permits others to distribute, remix, adapt, build upon this work non-commercially, and license their derivative works on different terms, provided the original work is properly cited, appropriate credit is given, any changes made indicated, and the use is non-commercial. See: http://creativecommons.org/licenses/by-nc/4.0/.

\section{REFERENCES}

1 Tyynismaa $\mathrm{H}$, Sun R, Ahola-Erkkilä S, et al. Thymidine kinase 2 mutations in autosomal recessive progressive external ophthalmoplegia with multiple mitochondrial DNA deletions. Hum Mol Genet 2012;21:66-75.

2 Garone C, Taylor RW, Nascimento A, et al. Retrospective natural history of thymidine kinase 2 deficiency. J Med Genet 2018;55:515-21.

3 Domínguez-González C, Hernández-Laín A, Rivas E, et al. LateOnset thymidine kinase 2 deficiency: a review of 18 cases. Orphanet J Rare Dis 2019;14:100.

4 Wang J, Kim E, Dai H, et al. Clinical and molecular spectrum of thymidine kinase 2-related mtDNA maintenance defect. Mol Genet Metab 2018;124:124-30.

5 De Troyer A, Borenstein S, Cordier R. Analysis of lung volume restriction in patients with respiratory muscle weakness. Thorax 1980;35:603-10.

6 Johnson EM, Roberts M, Mozaffar T, et al. Pulmonary function tests (maximum inspiratory pressure, maximum expiratory pressure, vital capacity, forced vital capacity) predict ventilator use in late-onset Pompe disease. Neuromuscul Disord 2016;26:136-45.

7 Morgan RK, McNally S, Alexander M, et al. Use of Sniff nasalinspiratory force to predict survival in amyotrophic lateral sclerosis. Am J Respir Crit Care Med 2005;171:269-74.

8 Andersen PM, Borasio GD, Dengler R, et al. EFNS Task force on management of amyotrophic lateral sclerosis: guidelines for diagnosing and clinical care of patients and relatives. Eur $J$ Neurol 2005;12:921-38.

9 Lopez-Gomez C, Levy RJ, Sanchez-Quintero MJ, et al. Deoxycytidine and deoxythymidine treatment for thymidine kinase 2 deficiency. Ann Neurol 2017;81:641-52.

10 Lopez-Gomez C, Hewan H, Sierra C, et al. Bioavailability and cytosolic kinases modulate response to deoxynucleoside therapy in TK2 deficiency. EBioMedicine 2019;46:356-67.

11 Blázquez-Bermejo C, Molina-Granada D, Vila-Julià F, et al. AgeRelated metabolic changes limit efficacy of deoxynucleosidebased therapy in thymidine kinase 2-deficient mice. EBioMedicine 2019;46:342-55.

12 Domínguez-González C, Madruga-Garrido M, Mavillard F, et al. Deoxynucleoside therapy for thymidine kinase 2-deficient myopathy. Ann Neurol 2019;86:293-303.

13 Ferguson GT, Enright PL, Buist AS, et al. Office spirometry for lung health assessment in adults: a consensus statement from the National lung health education program. Respir Care 2000;45:513-30.

14 Farrero E, Antón A, Egea CJ, et al. Guidelines for the management of respiratory complications in patients with neuromuscular disease. Sociedad Española de Neumología Y Cirugía Torácica (SEPAR). Arch Bronconeumol 2013;49:306-13.

15 Morelot-Panzini C, Bruneteau G, Gonzalez-Bermejo J. NIV in amyotrophic lateral sclerosis: The 'when' and 'how' of the matter. Respirology 2019;24:521-30. 